\title{
ARTÍCULOS DE REVISIÓN
}

doi: 10.11144/Javeriana.umed57-3.mnfi

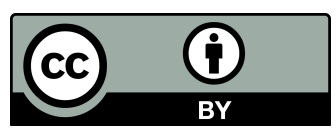

\section{Manejo no farmacológico del insomnio}

\author{
Gabriel Fernando Oviedo Lugo ${ }^{1}$, Patrick Rolando Verhelst Forero ${ }^{2}$, \\ VALERIA JORDAN MONDRAGÓN ${ }^{3}$
}

Cómo citar: Oviedo Lugo GF, Verhelst Forero PR, Jordan Mondragón V. Manejo no farmacológico del insomnio. Univ Med. 2016;57(3):348-66. doi: http://dx.doi.org/10.11144/Javeriana.umed57-3.mnfi

\begin{abstract}
Resumen
El insomnio es el trastorno de sueño más frecuente y, a menudo, se encuentra asociado a otros trastornos mentales. El diagnóstico se realiza cuando la queja principal es un sueño no reparador o una dificultad para iniciar o mantener el sueño y dura, al menos, tres meses. El objetivo del presente artículo es describir los diferentes modelos del insomnio primario, así como las aproximaciones terapéuticas no farmacológicas derivadas de estos esquemas. Las modalidades más comunes de tratamiento incluyen enfoques de tipo comportamental, control de estímulos, restricción de sueño, higiene de sueño y relajación muscular progresiva. Al ser el insomnio una condición crónica, se recomiendan tratamientos a largo plazo con un alto perfil de seguridad.
\end{abstract}

Palabras clave: sueño, insomnio, trastornos del inicio y mantenimiento del sueño, terapia cognitiva.

\section{Title: Non-Pharmacological Treatment of Insomnia}

\begin{abstract}
Insomnia is the most frequent sleep disorder and is often associated with psychiatric disorders. This diagnosis is made when the predominant complaint is difficulty initiating or maintaining sleep, or non-restorative sleep, for at least 3 months. The objective of this article is to describe the different models of insomnia and the non-pharmacologic therapeutic approaches. The most common therapy modalities include behavioral approaches, stimulus control, sleep restriction, sleep hygiene and
\end{abstract}

1 Médico psiquiatra, subespecialista en Psiquiatría de Enlace. Profesor del Departamento de Psiquiatría y Salud Mental, Pontificia Universidad Javeriana-Hospital Universitario San Ignacio, Bogotá, Colombia.

2 Médico psiquiatra. Fellow en Psiquiatría de Enlace, Pontificia, Universidad Javeriana, Bogotá, Colombia.

3 Médica psiquiatra, subespecialista en Psiquiatría de Enlace, Pontificia Universidad Javeriana, Bogotá, Colombia.

Recibido: 01/12/2015

Revisado: 22/01/2016

Aceptado: 11/02/2016 
progressive muscle relaxation. Since insomnia is a chronic condition, long-term and safe treatments are recommended.

Key words: sleep, insomnia, sleep initiation and maintenance disorders, cognitive-behavior therapy.

El sueño - un componente fundamental de la conducta humana - puede verse afectado por varios factores fisiológicos y patológicos, como medicamentos, diversas enfermedades o cambios propios del ciclo vital. Los trastornos primarios de sueño no se relacionan con un trastorno psiquiátrico, una afección médica o una sustancia; se infiere que su etiología está vinculada con alteraciones de los mecanismos que regulan el sueño y la vigilia exacerbados por factores de condicionamiento. La quinta versión del Manual diagnóstico y estadístico de los trastornos mentales (DSM-5) ha agrupado los trastornos de sueñovigilia en diez: trastorno de insomnio, trastorno de hipersomnia, narcolepsia, trastornos del sueño relacionados con la respiración, trastornos del ritmo circadiano del sueño-vigilia, parasomnias (que incluyen el trastorno del despertar del sueño no REM), trastorno de pesadillas, trastorno del comportamiento del sueño REM, síndrome de piernas inquietas y trastorno del sueño inducido por sustancias/medicamentos [1-3]. La Clasificación Internacional de los Trastornos de Sueño (ICSD-3) [4] ha reducido las categorías diagnósticas en comparación de la ICSD-2; pero esta nosología continúa generando un alto grado de especificidad en la subtipificación del insomnio (tabla 1) en contraste con el DSM-5 y la décima versión de la Clasificación Internacional de Enfermedades (CIE-10) [2,3,5].

Tabla 1. Resumen de las categorías diagnósticas del insomnio según la ICSD-3

\begin{tabular}{|l|l|}
\hline \multirow{4}{*}{ Insomnios } & Insomnio crónico \\
\cline { 2 - 2 } & Insomnio de corta duración \\
\cline { 2 - 2 } & Otros insomnios \\
\hline
\end{tabular}

El insomnio es el más frecuente de todos los trastornos de sueño en la población general [1]. Se entiende por este la dificultad persistente para mantener y conciliar el sueño, despertar precoz o sueño no reparador. Lichstein y colaboradores definen el insomnio desde un punto de vista cuantitativo, como una latencia de inicio de sueño o un tiempo de despertar mayor a 31 minutos que ocurre al menos 3 veces a la semana, al menos por un periodo de 6 meses. [6]

Los criterios del DSM-5 para insomnio primario (tabla 2) muestran que su duración resulta ser un factor importante para su orientación. De acuerdo con esta, se considera insomnio episódico cuando los síntomas duran, como mínimo, un mes, pero menos de tres meses; insomnio persistente, cuando lo síntomas durante tres meses o más, e insomnio recurrente cuando ocurren dos 
o más episodios en el curso de un año $[7,6]$. De acuerdo con su modo de presentación en otras clasificaciones, este puede ser de conciliación (dificultad del paciente para quedarse dormido), de mantenimiento (cuando el sueño se ve interrumpido a lo largo de la noche y hay dificultad para reconciliarlo) y de despertar temprano (frecuente en ancianos y pacientes con depresión). Se denomina insomnio paradójico a una mala percepción del propio sueño, y la característica principal es una queja de insomnio, sin que pueda documentarse un trastorno de tal magnitud mediante la realización de pruebas diagnósticas como el polisomnograma [8-10].

\section{Epidemiología}

El insomnio es una queja bastante prevalente en la población general y quizás es el trastorno más frecuente dentro del grupo que conforma el trastorno del sueño. En algún momento de la vida, hasta un $50 \%$ de los adultos lo pade-

Tabla 2. Trastorno de insomnio: criterios del DSM-5

\begin{tabular}{|l|l|}
\hline A. & $\begin{array}{l}\text { Predominante insatisfacción por la cantidad o la calidad del sueño asociada a uno } \\
\text { (o más) de los síntomas siguientes: 1) dificultad para inicial el sueño; 2) dificultad } \\
\text { para mantener el sueño que se caracteriza por despertares frecuentes y problemas } \\
\text { para volver a conciliar el sueño después de despertar; 3) despertar pronto por la } \\
\text { mañana con incapacidad para volver a dormir. }\end{array}$ \\
\hline B. & $\begin{array}{l}\text { La alteración del sueño (o la fatiga diurna asociada) provoca malestar clínicamente } \\
\text { significativo o deterioro social, laboral o de otras áreas importantes del funcio- } \\
\text { namiento. }\end{array}$ \\
\hline C. & La dificultad del sueño se produce al menos tres noches a la semana. \\
\hline D. & La dificultad del sueño está presente durante un mínimo de tres meses. \\
\hline E. & $\begin{array}{l}\text { La dificultad del sueño se produce a pesar de las condiciones favorables para } \\
\text { dormir. }\end{array}$ \\
\hline F. & $\begin{array}{l}\text { El insomnio no se explica mejor por otro trastorno del sueño-vigilia y no se } \\
\text { produce exclusivamente en el curso de otro trastorno del sueño-vigilia (p. ej., } \\
\text { narcolepsia, un trastorno del sueño relacionado con las respiración, un trastorno } \\
\text { del ritmo circadiano del sueño-vigilia, una parasomnia). }\end{array}$ \\
\hline G. & $\begin{array}{l}\text { El insomnio no se puede atribuir a los efectos fisiológicos de una sustancia (p. ej., } \\
\text { una droga, un medicamento). }\end{array}$ \\
\hline H. & $\begin{array}{l}\text { La coexistencia de trastornos mentales y afecciones médicas no explica adecuada- } \\
\text { mente la presencia predominante de insomnio. }\end{array}$ \\
\hline Especificar si: & $\begin{array}{l}\text { Con trastorno mental concurrente no relacionado con el sueño, incluidos los } \\
\text { trastornos por consumo de sustancias. } \\
\text { Con otra afección médica concurrente. } \\
\text { Con otro trastorno de sueño. }\end{array}$ \\
\hline Especificar si: & $\begin{array}{l}\text { Episódico: los síntomas duran como mínimo un mes; pero menos de tres meses. } \\
\text { Persistente: los síntomas duran tres meses o más. } \\
\text { Recurrente: dos (o más) episodios den el plazo de un año. }\end{array}$ \\
\hline
\end{tabular}


cen ante situaciones estresantes. Del $10 \%$ al $20 \%$ de las personas que presentan esta condición son afectados por ella crónicamente [11-13]. El insomnio persistente conlleva una elevada carga para la sociedad, como se evidencia en los índices de funcionalidad, calidad de vida, riesgo de depresión y costos en salud [13-16].

En un reciente estudio llevado a cabo en Estados Unidos, que buscaba evaluar los motivos de consulta asociados a trastornos del sueño durante 10 años, el número de visitas al consultorio con insomnio como la razón aducida para la visita aumentó de 4,9 millones de visitas en 1999 a 5,5 millones de visitas en 2010, con un incremento del $13 \%$ [17].

Las mujeres son más susceptibles a presentar quejas de insomnio en relación con los hombres (el $40 \%$ de las mujeres se queja de insomnio en contraste con un $30 \%$ de los hombres). $\mathrm{Al}$ evaluarse personas mayores de 65 años de edad, una de cada dos se queja de insomnio y una tercera parte de los adultos tiene insomnio crónico. Aproximadamente un $20 \%$ de los pacientes reporta alteraciones del sueño significativas en atención primaria $[1,11,15]$.

Al evaluarse otras características demográficas, como estatus marital, nivel socioeconómico, educación, tipo de trabajo y estresores asociados a lo laboral, la mayoría de los estudios sugiere que los viudos, los solteros o los divorciados tienen mayor riesgo de presentar insomnio, comparados con los individuos casados. Otros estudios muestran cómo los desempleados son proclives a desarrollarlo. En los grupos de empleados, las personas que realizan rotaciones $o$ turnos en las noches presentan mayor riesgo de insomnio $[9,15]$.

Varios factores comportamentales y ambientales aumentan el riesgo de insomnio como un estilo de vida estresante, inactividad física, siestas irregulares, dependencia al alcohol y uso de cafeína. La alteración crónica de sueño que caracteriza al insomnio primario constituye un factor de riesgo para la aparición posterior de un trastorno de ansiedad o un trastorno depresivo mayor. Se estima que de un $35 \%$ a un $40 \%$ de los pacientes con insomnio tiene una o más comorbilidades asociadas a un diagnóstico psiquiátrico. Por otro lado, se considera que el $75 \%$ de los pacientes enfermos psiquiátricos presenta dificultades para dormir en la fase aguda de la enfermedad [9]. Entre los individuos con trastornos médicos no psiquiátricos o psiquiátricos crónicos el insomnio es aún más común y puede impedir la recuperación de la enfermedad [18].

Las quejas de mal dormir son frecuentes, pero pocos pacientes solicitan atención sanitaria de forma específica. Esta falta de atención médica contrasta con la elevada utilización de fármacos 
para tratarlas, fundamentalmente benzodiacepinas. Se ha demostrado que la mitad de los pacientes que toma benzodiacepinas diariamente durante más de 6 meses había iniciado el tratamiento por quejas de insomnio [11].

Desde el punto de vista económico, el insomnio tiene un fuerte impacto para la sociedad actual, pues a los costos directos derivados de su diagnóstico y tratamiento, se deben agregar los costos indirectos asociados a la morbilidad y mortalidad asociadas como mayores índices de ausentismo laboral y a una mayor utilización de los servicios de salud; concomitantemente, se asocia un menor nexo con las relaciones interpersonales y mayor riesgo de accidentes laborales y de tránsito $[19,20]$. A pesar de su alta prevalencia y el impacto negativo, el insomnio permanece subdiagnosticado y no tratado en aproximadamente un $80 \%$ de los casos. En general, cuando el médico de atención primaria recibe casos de insomnio, su tratamiento se limita a la administración de medicación hipnótica. Debemos tener en cuenta que los efectos de tolerancia, dependencia psicológica e insomnio iatrogénico por el uso continuado de hipnóticos y sedantes plantea un problema grave $[1,7,19]$.

\section{Modelo psicofisiológico del insomnio}

El modelo fisiológico sugiere que el insomnio crónico se entiende como una condición en la que el paciente tiene un grado de alertamiento previo o durante el periodo preferido para dormir, incompatible con una continuidad de sueño adecuada [21-23]. Es sabido que el sueño humano se regula por la interacción de tres sistemas neurales mayores: uno homeostático, que incrementa la posibilidad de sueño en relación con las horas de vigilia; un proceso circadiano, que genera un ritmo biológico de tendencia sueño-despertar sobre el día, y un sistema de alertamiento, que promueve el estado de alerta en oposición a la facilitación para dormir $[7,21]$. Varios estudios han demostrado un elevado alertamiento fisiológico en las personas con insomnio, comparadas con personas con adecuado patrón de sueño basado en medidas electrofisiológicas, como frecuencia cardiaca y su variabilidad, frecuencia respiratoria, temperatura corporal, tono muscular, conductancia cutánea y resistencia vascular periférica [24,25]. Se mostró también un aumento en el procesamiento cognitivo alrededor del inicio del sueño, hecho demostrado por frecuencias electroencefalográficas más rápidas [26]. La activación del eje hipotálamohipofisiario-adrenal aporta mayor evidencia acerca de las implicaciones de una respuesta en un sistema de estrés. Medidas urinarias de 11 hidroxicosteroides libres en 24 horas de personas jóvenes con insomnio comparadas con controles sin insomnio son significativamente mayores en las personas con insomnio [27]. Se han encontrado más 
concentraciones de ACTH y cortisol en el curso de un día en un grupo de insomnes, comparado con controles sanos [28].

Así, al integrar los anteriores conceptos, podemos ver que las dificultades para dormir se fundamentan en un impulso homeostático débil, una regulación circadiana atípica o un rasgo de hiperalertamiento constitucional.

\section{Modelos cognitivos de insomnio}

Los modelos cognitivos del insomnio sugieren que este ocurre en asociación con un estado de hiperalertamiento, donde el sueño y el hiperalertamiento son mutuamente excluyentes. Se ha demostrado una asociación significativa entre cogniciones negativas alrededor del sueño y una mala calidad de sueño o insomnio. La tendencia a la rumiación y a la preocupación sirven como facilitadores del insomnio.

Varios individuos creen que su mala calidad de sueño es inevitable y centran sus preocupaciones en esta condición, por lo que todos sus problemas están asociados a ello [29-31]. Además, esta preocupación y rumiación sirve como un factor perpetuador del insomnio, en la medida en que se convierte en un círculo vicioso que da como resultante una falta de sueño que refuerza su propia creencia.
La catastrofización es un tercer proceso cognitivo que viene recibiendo más atención en los últimos años, y se define como la composición catastrófica de pensamientos que llegan a ser un factor que contribuye al insomnio, ya que su presencia aumenta la ansiedad y el malestar y, así, se genera una actividad cognitiva que conduce a la hipervigilancia [31]. Las cogniciones disfuncionales alrededor del sueño pueden generar un comportamiento maladaptativo que lleva a largas horas de permanencia en la cama, que alteran el ritmo circadiano y que llevan a periodos prolongados de despertar (figura 1).

Es frecuente que un individuo bajo un estrés emocional agudo encuentre dificultades para dormir, pues el sistema nervioso autónomo y el sistema hormonal reaccionan a este tipo de emociones. Estos procesos fisiológicos alteran los mecanismos normales de sueño, y así es como trazas de insomnio transitorio pueden resultar de una experiencia estresante aguda; pero algunos individuos son más susceptibles a la cronificación de este síntoma. Mauss y colaboradores asocian una peor calidad del sueño con una disminución en la capacidad de regular las emociones negativas mediante el uso de herramientas de reevaluación cognitiva [23].

Estudios de personalidad en insomnes muestran individuos que tienden a 


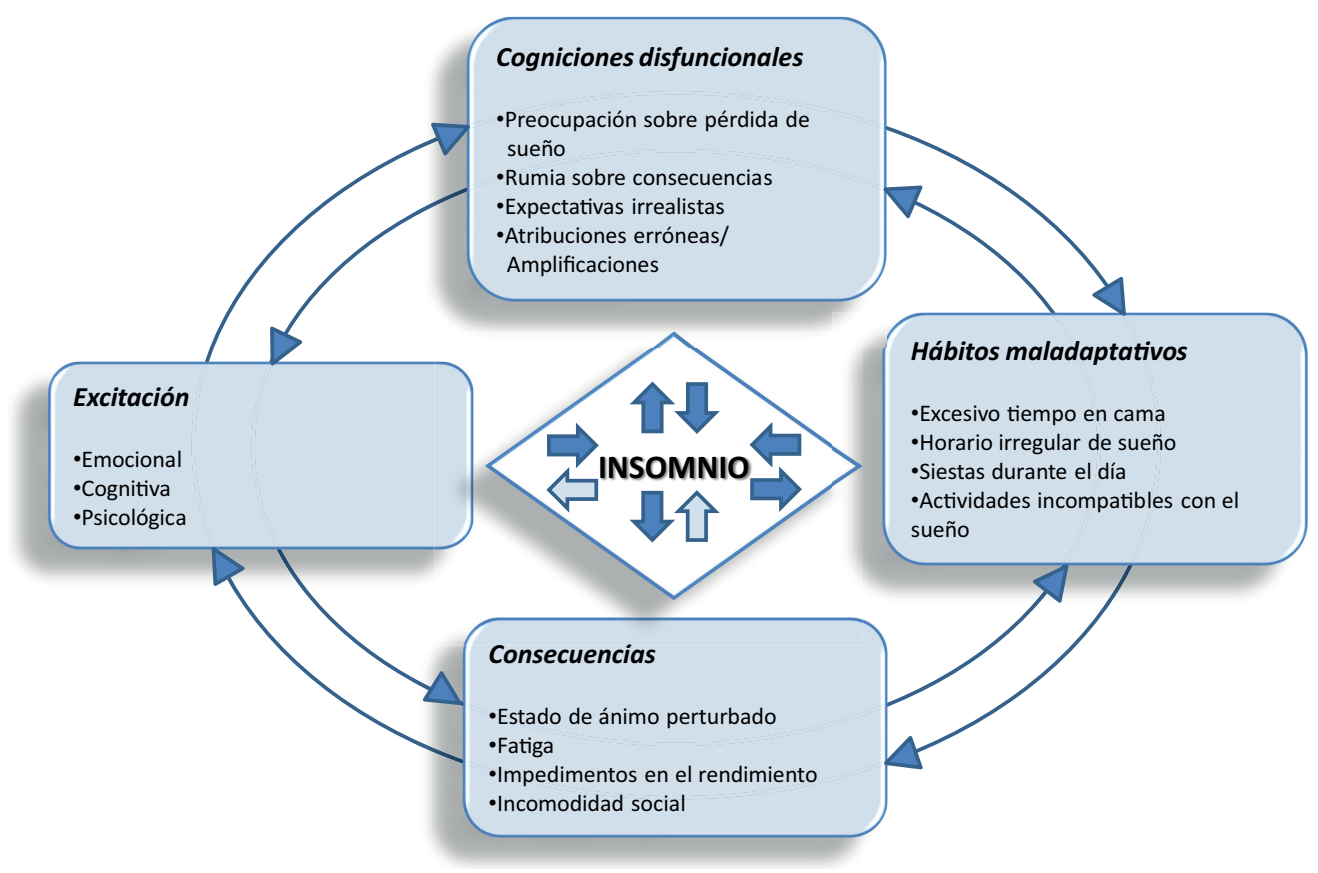

Figura 1. Modelo cognitivo-comportamental del insomnio crónico

Fuente: adaptado de Morin CM. Insomnia: psychological assesment and management. New York: Guilford Press; 1993. p. 57.

interiorizar conflictos a través de inhibición o negación, así como tendencias perfeccionistas y necesidad de control [27]. Harvey [32] ha propuesto que la naturaleza autoperpetuadora del insomnio se relaciona con la preocupación acerca del dormir, lo cual puede afectar un proceso cognitivo y de comportamiento que media la ocurrencia y la severidad del insomnio crónico. De acuerdo con este modelo, el insomnio agudo se presenta en asociación con estresores vitales, el insomnio subcrónico ocurre con preocupaciones acerca del dormir y el insomnio crónico se mantiene por atención selectiva y automonitoreo permanente [21,22].

\section{Modelos comportamentales del insomnio}

La perspectiva comportamental sugiere que aun cuando una variedad de factores biopsicosociales puede precipitar el insomnio agudo, el crónico resulta de comportamientos que perturban el sueño (figura 1). Se ha descrito un modelo de control de estímulos basado en el principio comportamental de que un estímulo puede evocar una serie de respuestas dependiendo de la historia de condicionamiento. A menudo, en personas con insomnio, las condiciones habituales relacionadas con el sueño (habitación, hora de acostarse) son 
asociadas con actividades diferentes al dormir.

Glovinski y colaboradores [21] y Yang y colaboradores [22] introdujeron el modelo de las 3P, que agrupa factores etiológicos del insomnio en predisponentes, características precipitantes y prácticas o actitudes perpetuadoras. A menudo, las características predisponentes se presentan durante varios años antes del inicio del insomnio, referente a muchas tendencias relacionadas con el hiperalertamiento o una propensión innata a preferir actividades en la noche, personalidades más ansiosas, historia familiar o personal de insomnio, edad y sexo (es más común en las mujeres). Existen también factores predisponentes adquiridos, por ejemplo, trastornos de dolor residual, que no siempre están acompañados por insomnio crónico, pero pueden disminuir el umbral para la aparición de este trastorno. Los factores precipitantes son eventos recientes que interrumpen el sueño, son de tipo "gatillo" y se tratan generalmente de estresores de tipo vital (por ejemplo, enfermedades, hospitalizaciones o separaciones); amenazas menos graves, pero más crónicas (por ejemplo, estrés ocupacional o conflictos familiares), $\mathrm{u}$ otros cambios importantes de la vida (por ejemplo, nacimiento de un hijo o menopausia). Los factores perpetuadores se refieren a estrategias que el paciente adopta para compensar la pérdida de sueño, como ir a la cama más temprano o levantarse más tarde, realizar siestas. Estas estrategias llevan generalmente a un desbalance entre la oportunidad de sueño y la habilidad de sueño.

\section{Modelo neurocognitivo del insomnio}

El modelo neurocognitivo considera el hiperalertamiento dentro de tres dimensiones confluentes: la somática, la cognitiva y la cortical, que se enfocan en la medida de las consecuencias del alertamiento cortical condicionado. Se cree que el alertamiento cortical es resultado del condicionamiento clásico y puede observarse en pacientes con insomnio primario, como una alta frecuencia de la actividad electroencefalográfica cerca al momento de dormir y durante el sueño NREM. También se ha demostrado que los individuos con insomnio paradójico exhiben mayor actividad beta. Los análisis correlacionales muestran cómo está negativamente asociada con la percepción de la calidad de sueño. Este modelo integra, desde una perspectiva neurobiológica, varias consideraciones asociadas con la teoría del aprendizaje y contribuye al entendimiento de la patogénesis del insomnio. Las limitaciones de este modelo tienen que ver con la falta de consideración de la importancia de las influencias circadianas y homeostáticas en el sueño $[26,33]$. 


\section{Evaluación}

La evaluación de la queja de insomnio se fundamenta en la entrevista clínica; pero puede ser complementada por una serie de metodologías accesorias. Los elementos fundamentales de la evalua- ción del paciente con queja de sueño se resumen en la tabla 3.

Cabe anotar que dentro del enfoque del paciente con queja de sueño, aparte de la consideración diagnóstica de

Tabla 3. Componentes de la evaluación clínica de insomnio

\begin{tabular}{|c|c|}
\hline \multirow{14}{*}{ Historia de sueño } & $\begin{array}{l}\text { Naturaleza de la queja (patrón, inicio, historia, curso, duración, } \\
\text { severidad) }\end{array}$ \\
\hline & Factores predisponentes y precipitantes \\
\hline & Factores que exacerban el insomnio o mejoran el patrón de sueño \\
\hline & Factores etiológicos \\
\hline & Patrón del ciclo sueño-vigilia \\
\hline & Síntomas diurnos (hipersomnia o hiperalertamiento) \\
\hline & Impacto en la vida diaria \\
\hline & Acondicionamiento al ambiente de la habitación \\
\hline & Creencias acerca del sueño y causas del insomnio \\
\hline & Síntomas acerca de otros trastornos de sueño \\
\hline & $\begin{array}{l}\text { Ambiente de sueño (rutinas a la hora de acostarse, comportamien- } \\
\text { tos incompatibles con el sueño) }\end{array}$ \\
\hline & Estilo de vida \\
\hline & $\begin{array}{l}\text { Historia de tratamiento (técnicas de autoayuda, estrategias de afron- } \\
\text { tamiento, respuesta a tratamientos previos) }\end{array}$ \\
\hline & Expectativas acerca del tratamiento \\
\hline \multirow{5}{*}{$\begin{array}{l}\text { Uso de medicamentos y } \\
\text { sustancias }\end{array}$} & Medicamentos, remedios caseros y herbales \\
\hline & Medicamentos prescritos \\
\hline & Medicamentos de mostrador \\
\hline & Uso de alcohol, tabaco y cafeína \\
\hline & Sustancias de abuso \\
\hline \multirow{5}{*}{ Antecedentes y exámenes } & Trastornos médicos asociados con alteraciones del sueño \\
\hline & Dolor crónico \\
\hline & Menopausia \\
\hline & Enfermedad prostática \\
\hline & Exámenes de laboratorio \\
\hline \multirow{4}{*}{ Historia psiquiátrica } & Trastornos afectivos \\
\hline & Trastorno de ansiedad \\
\hline & Niveles de estrés \\
\hline & Otros trastornos mentales \\
\hline
\end{tabular}


insomnio primario, se debe explorar la posibilidad de la existencia de condiciones prevalentes como son los trastornos del ritmo circadiano de sueño-vigilia, los trastornos de sueño relacionado con la respiración y el síndrome de piernas inquietas [2].

La evaluación del insomnio se facilita con el uso de un diario de sueño utilizado por una o dos semanas. El paciente registra varios aspectos relacionados con el tiempo y su patrón de sueño, incluyendo latencias, distribución y duración de los episodios de sueño, número de despertares, tiempos de vigilia y siestas. La historia clínica debe incluir una revisión por sistemas de aspectos patológicos comúnmente relacionados con el insomnio como enfermedades reumatológicas, pulmonares, cardiacas, gastrointestinales, neurológicas, endocrinas, así como una historia psiquiátrica. Eventos relacionados con el consumo de cafeína y alcohol, ejercicio, exposición a la luz y medicamentos también son registrados $[16,20]$.

Los diarios de sueño proveen información más confiable acerca de la severidad y la variabilidad del insomnio que el reporte retrospectivo del paciente. Si la historia clínica sugiere la posibilidad de condiciones fisiológicas subyacentes asociadas a la disrupción de sueño como un trastorno relacionado con la respiración o un trastorno del comportamiento del sueño REM, debe ser referido para un estudio videopolisomnográfico $[10,16,20]$.

\section{Intervenciones}

A pesar de la alta prevalencia y la carga que este conlleva, el insomnio viene en gran parte subdiagnosticado $\mathrm{y}$, por lo tanto, cursa sin un tratamiento adecuado. La mayoría de los tratamientos encontrados, en gran parte de tipo farmacológico, son automedicados y no consideran sus efectos secundarios. Cuando llega la queja de insomnio al médico tratante, existe la tendencia a utilizar fármacos hipnóticos, y aunque estos tienen una indicación clara en el insomnio, los factores psicológicos y comportamentales son casi siempre factores perpetuadores de los trastornos del sueño, y estos deben ser explorados especialmente en los casos de insomnio crónico [7].

En la última década se han realizado importantes avances sobre las intervenciones no farmacológicas del insomnio; pero aunque este tipo de tratamientos son muy bien aceptados por parte de los pacientes, su conocimiento médico es muy pobre y por lo tanto subutilizados. Los objetivos del tratamiento del insomnio son mejorar los aspectos cuantitativos y cualitativos de sueño, reducir la angustia y la ansiedad asociados con la falta de sueño y mejorar el rendimiento durante el día [12]. 
Dentro de las terapias cognitivascomportamentales se incluyen la terapia de control de estímulos, la terapia restricción de sueño, el entrenamiento en relajación, la terapia cognitiva, la educación en higiene de sueño y la terapia cognitivo-conductual combinada (TCC) (tabla 4).

\section{Terapia cognitivo-conductual}

La TCC es una intervención multimodal que incluye un componente terapéutico para abordar los factores psicológicos y cognitivos asociados (creencias, expectativas, aprehensiones y preocupaciones) y un componente más específico hacia los factores comportamentales (hábitos de sueño maladaptativos, horarios irregulares de sueño) que se presume cumplan un papel de perpetuadores del insomnio. Por lo tanto, el objetivo principal de la TCC es alterar esos factores que exacerban los trastornos del sueño: pobres hábitos de sueño (por ejemplo, estar mucho tiempo en la cama), horarios de sueño-vigilia irregulares (por ejemplo, levantarse a horas diferentes), hiperalertamiento, higiene del sueño inadecuado, y los conceptos erróneos alrededor del sueño y de las consecuencias del insomnio. Otra meta de este tipo de intervención es enseñar al paciente a reconocer y poder utilizar las maniobras aprendidas para adaptarse a aquellas manifestaciones residuales que pueden presentarse después de la terapia. En el tratamiento se realizan de
Tabla 4. Resumen de las intervenciones psicológicas y comportamentales para el manejo del insomnio primario

\begin{tabular}{|c|c|}
\hline $\begin{array}{l}\text { Terapia de } \\
\text { control de } \\
\text { estímulos }\end{array}$ & $\begin{array}{l}\text { Serie de instrucciones dise- } \\
\text { ñadas para reasociar la cama- } \\
\text { habitación con el sueño y } \\
\text { restablecer un horario con- } \\
\text { sistente de sueño vigilia. Ir a } \\
\text { la cama solo cuando se tiene } \\
\text { sueño, salir de la cama cuando } \\
\text { no sea capaz de dormir, utili- } \\
\text { zar la cama solo para dormir y } \\
\text { la actividad sexual. }\end{array}$ \\
\hline $\begin{array}{l}\text { Terapia } \\
\text { restricción } \\
\text { de sueño }\end{array}$ & $\begin{array}{l}\text { Método por el cual se crea una } \\
\text { leve deprivación de sueño que } \\
\text { resulta en un sueño consolida- } \\
\text { do y más eficiente. }\end{array}$ \\
\hline $\begin{array}{l}\text { Entrenamiento } \\
\text { en relajación }\end{array}$ & $\begin{array}{l}\text { Procedimientos orientados a } \\
\text { reducir la tensión somática } \\
\text { (relajación muscular progresi- } \\
\text { va, entrenamiento autógeno) o } \\
\text { pensamientos intrusivos (me- } \\
\text { ditación) que interfieren con } \\
\text { el sueño. }\end{array}$ \\
\hline $\begin{array}{l}\text { Terapia } \\
\text { cognitiva }\end{array}$ & $\begin{array}{l}\text { Método psicoterapéutico orien- } \\
\text { tado a cambiar creencias dis- } \\
\text { torsionadas y actitudes acerca } \\
\text { del sueño, el insomnio y las } \\
\text { consecuencias del día siguien- } \\
\text { te. Otras estrategias cognitivas } \\
\text { se usan para controlar pensa- } \\
\text { mientos intrusitos a la hora de } \\
\text { acostarse y prevenir un moni- } \\
\text { toreo excesivo de las conse- } \\
\text { cuencias del insomnio. }\end{array}$ \\
\hline $\begin{array}{l}\text { Educación } \\
\text { en higiene } \\
\text { de sueño }\end{array}$ & $\begin{array}{l}\text { Guías generales acerca de } \\
\text { prácticas saludables (dieta, } \\
\text { ejercicio, uso de sustancias), } \\
\text { y factores ambientales (luz, } \\
\text { ruido, temperatura) que pue- } \\
\text { den promover o interferir el } \\
\text { sueño. }\end{array}$ \\
\hline
\end{tabular}


4 a 10 sesiones generalmente estructuradas con una duración aproximada de 50 a 60 minutos una vez a la semana, con un espacio de 2 semanas para permitir la automonitorización de conductas relacionadas con el sueño [13,34]. En una revisión de la literatura, Mitchell y colaboradores [13] encontraron que la TCC es un tratamiento eficaz para el insomnio y que puede producir resultados duraderos en un relativamente breve número de sesiones. Algunos hallazgos sugieren que la TCC tiene una mayor eficacia que los medicamentos para el tratamiento del insomnio 6 meses o más después de finalizar el tratamiento y un mayor impacto en la funcionabilidad del paciente. La indicación principal de la TCC es el tratamiento del insomnio persistente, tanto primario como secundario [21,26,29,34-37].

\section{Terapia de restricción del sueño}

Aquellos que padecen de alguna alteración del sueño tienden a aumentar el tiempo en la cama en busca de la conciliación de este. Ello usualmente lleva a un sueño fragmentado y de poca calidad. La restricción del sueño consiste en reducir la cantidad de tiempo gastado en la cama a la cantidad real de tiempo dormido. Es decir, si una persona duerme aproximadamente 6 horas de las 8 horas en las que se encuentra en la cama, entonces la ventana de sueño (el tiempo desde que se acuesta hasta que se levanta) se encuadra en 6 horas. Los ajustes posteriores se realizan de acuerdo con la eficiencia del sueño (ES: [tiempo de sueño/tiempo en cama] $\times 100$ ) por un periodo determinado (usualmente el de la última semana); se aumenta el tiempo en la cama de 20 minutos por semana si la ES supera el $85 \%$, y se disminuye de igual manera si la ES es inferior al $80 \%$. La ES se mantiene estable cuando se encuentra entre el $80 \%$ y $85 \%$.

Los cambios que se realicen a esta ventana pueden asignarse tanto en las noches (acostarse más tarde) como en las mañanas (levantarse más temprano). Para evitar efectos colaterales como la somnolencia diurna, el sueño no debe ser reducido de 5 horas de sueño y se deben dar las advertencias pertinentes como no manipular o realizar actividades que requieran una total hipervigilancia. La finalidad de este tipo de terapia es buscar un sueño más eficiente y disminuir así la ansiedad anticipatoria. Esta terapia puede ser útil sola o en combinación con otros tipos de terapias cognitivo-comportamentales [21,22,29,36,38,39].

\section{Terapia de control de estímulos}

Con gran frecuencia, los individuos que padecen de insomnio se tornan temerosos llegada la hora de acostarse, pues asocian la habitación con frustración y múltiples despertares, más que con sueño. Este tipo de terapia consiste en una serie de instrucciones para reasociar estímulos ambientales (la cama y la habi- 
tación) y temporales (hora de acostarse) con una conciliación más rápida y establecer un ritmo circadiano más regular. Las instrucciones son:

1. Acostarse con sueño.

2. Levantarse tras 20 minutos de insomnio.

3. Restringir actividades incompatibles con el sueño.

4. Hora de despertarse.

5. No tomar siestas.

En diferentes estudios controlados se ha demostrado la eficacia de esta terapia tanto para el insomnio de conciliación como para el de múltiples despertares; así mismo, se ha estudiado su uso en conjunto con otras terapias $[10,21,22,35,36]$.

\section{Entrenamiento en relajación}

Quizás el entrenamiento en relajación sea la terapia más utilizada aparte de la terapia farmacológica. De las diferentes intervenciones de relajación, algunos métodos se enfocan principalmente en reducir la tensión somática (relajación autógena y relajación progresiva), y otras más en la reducción de pensamientos intrusivos (imaginería y meditación). La mayoría de estas intervenciones son igualmente eficaces para el insomnio. El punto más crítico está en regular su práctica en, por lo menos, dos a cuatro semanas. Este método re- quiere un seguimiento inicial por algún entrenador; pero es muy útil en personas con un gran monto de ansiedad y tensión. Además, es necesario tener algunas precauciones, dado que algunos pacientes pueden tener una respuesta paradójica y aumentar la ansiedad [10]. En diferentes ocasiones la relajación se ha asociado a la TCC; sin embargo, algunos estudios han demostrado que la relajación, por sí misma, es más eficiente que en combinación $[30,40]$.

\section{Terapia cognitiva}

La terapia cognitiva es un método psicoterapéutico que busca modificar las cogniciones disruptivas (creencias, expectativas y atribuciones) alrededor del sueño, así como aquellos procesos maladaptativos (automonitoreo excesivo, preocupaciones) a través de preguntas y experimentos comportamentales. La premisa básica de este tratamiento es que la sobreapreciación de una situación determinada y el exceso de automonitoreo pueden desencadenar una respuesta emocional que es incompatible con el sueño.

La terapia cognitiva está diseñada para identificar cogniciones disfuncionales y sustituirlas con cogniciones más adaptativas; por ello con esta reestructuración cognitiva se busca:

- Mantener expectativas realistas.

- No culpabilizar al insomnio. 
- No intentar dormir.

- No sobrevalorar el sueño.

- Evitar las ideas catastróficas tras poco sueño.

- Tolerancia ante el insomnio.

Usualmente, este tipo de terapia se encuentra dentro de una intervención de tipo cognitivo-comportamental, y no existen muchos estudios que demuestren su eficacia como terapia única para el manejo del insomnio. Un reciente metanálisis que usó la TCC como coadyuvante en el tratamiento de condiciones médicas-psiquiátricas y no psiquiátricas demostró que el uso de esta terapia fue efectivo en el manejo del insomnio asociado a otras enfermedades [18].

Harvey y colaboradores encontraron que la terapia cognitiva tardó más tiempo en ser efectiva; pero logró ser una intervención más beneficiosa en cuanto a resolución del insomnio, en comparación con la terapia conductual [34,36].

La intención paradójica es un tipo especial de intervención cognitiva, referente a instrucciones específicas para involucrar al paciente de manera consciente en los comportamientos que más teme, por ejemplo, quedarse despierto. A pesar de que existe evidencia que respalda este tipo de terapia en el insomnio inicial, su uso ha declinado [38].

\section{Meditación de atención plena: mindfulness}

La meditación de atención plena es una práctica de autorregulación con varios beneficios para la salud y que ha sido combinada con la TCC para el tratamiento del insomnio [41]. Integra elementos de las estrategias conductuales para el insomnio, es decir, la restricción de sueño y el control de estímulos con prácticas de meditación mindfulness. Una revisión detallada acerca de los modelos de intervención clínica basados en esta va más allá del alcance de este artículo.

Las prácticas de meditación de atención plena ayudan a las personas a aprender cómo enfocar la conciencia en el momento presente y a cambiar su relación con los pensamientos relacionados con el sueño, que tienden a ser una importante fuente de ansiedad para los pacientes que sufren de insomnio crónico. Los resultados de algunas investigaciones indican que la combinación de meditación de atención plena con las estrategias de la TCC conduce a una reducción significativa del tiempo total de vigilia en el autorreporte y la hiperactivación, que ha demostrado mantenerse hasta 12 meses postratamiento. No obstante, se necesitan más estudios para determinar si puede ser una alternativa viable a la TCC $[38,41]$. 


\section{Higiene del sueño}

La higiene del sueño se refiere a la noción de que tipos especiales de comportamiento llevan a condiciones incompatibles con el sueño y que la modificación comportamental asociada puede mejorar el insomnio [35,42]. Un estudio realizado por Gancedo y colaboradores [43] con respecto a la aplicación de pautas de higiene de sueño mostró la eficacia de estas en la mejoría de insomnio de los pacientes que las recibieron en un modelo psicoeducativo en el consultorio. Las pautas de higiene de sueño se resumen así:

1. Levantarse siempre a la misma hora.

2. Permanecer en la cama el tiempo acostumbrado antes del trastorno de sueño.

3. Suspender el consumo de sustancias con actividad en el sistema nervioso central (alcohol, nicotina, cafeína, estimulantes).

4. Evitar siestas durante el día.

5. Realizar un programa de ejercicio diario temprano o en la tarde.

6. Eliminar estímulos nocturnos, televisión, uso de computadores.

7. Intentar elevar la temperatura corporal mediante un baño caliente previo 20 minutos antes de dormir.
8. Comer diariamente a horas regulares evitando comidas pesadas cerca de la hora de acostarse.

9. Practicar rutinas relajantes en la noche como relajación muscular progresiva o meditación.

10. Mantener condiciones confortables para dormir.

Adicionalmente, es muy útil proporcionar información básica sobre el sueño normal, diferencias individuales en las necesidades del sueño y cambios fisiológicos a través del ciclo vital. Esta información ayuda a diferenciar entre el insomnio clínico y el insomnio dentro de un sueño normal. Estas instrucciones deben hacer parte de todo un programa de intervenciones al insomnio y no como método único.

\section{Actividad fisica}

Varios estudios han sugerido que la falta de sueño puede estar relacionada con inactividad física o con un estilo de vida sedentario. El ejercicio se considera una intervención no farmacológica eficaz para prevenir o reducir problemas del sueño. Este último y el ejercicio influyen entre sí a través de interacciones complejas que incluyen diversas vías fisiológicas y psicológicas. El ejercicio provoca cambios fisiológicos favorables en la regulación homeostática del sueño, lo que aumenta las ondas lentas (sueño profundo). 
La actividad física también tiene la capacidad de estabilizar el sistema circadiano y de disminuir la somnolencia. Los autorreportes de pacientes sugieren que hombres y mujeres consideran que el ejercicio es uno de los factores más importantes para fomentar el sueño; sin embargo, se ha cuestionado la causalidad de esta relación: aunque esta intervención, en general, se considera beneficiosa para el sueño, varios factores pueden afectar esta relación, incluidos el género, la edad, la condición física, la calidad del sueño y el tipo de ejercicio (intensidad, duración, hora del día, el medio ambiente) [38]. Un reciente estudio sobre la relación entre la actividad física y el ejercicio reportó que el de tipo aeróbico de intensidad moderada se recomienda en el tratamiento y la prevención de trastornos del sueño.

Hacer ejercicio antes de acostarse puede no generar siempre alteraciones en el patrón de sueño en quienes dormían bien previo a este problema; pero los efectos beneficiosos del ejercicio regular sobre el sueño pueden explicarse por múltiples factores: interacción del ritmo circadiano, metabólicos, inmunológicos, termorregulación, vasculares, estado de ánimo y endocrinos [44].

\section{Comentario final}

Dado que el insomnio es un problema recurrente, y los pacientes que se someten a tratamiento usualmente han pre- sentado insomnio durante varios años, es importante evaluar el desenlace más allá del inicio del tratamiento dos o tres meses después. Varios estudios han mostrado las mejorías presentadas con intervenciones de tipo TCC, las cuales se mantienen en el tiempo, con datos disponibles hasta de 24 a 36 meses posteriores al tratamiento [45]. Esta mejoría en el tiempo es una gran ventaja sobre la terapia farmacológica. Sin embargo, estos datos deben ser tomados en cuenta con precaución, dado que en literatura no hay seguimientos a largo plazo [46-50].

Dentro de las dificultades para tratar el insomnio con este tipo de terapias se resaltan el tiempo y los costos que implica someterse a estas, así como la limitada experiencia por parte de los clínicos en estos métodos. Diversos estudios han demostrado que parte del tratamiento también puede ser implementado por personal del grupo paramédico entrenado, como enfermeros y médicos de atención primaria.

\section{Referencias}

1. Ohayon M. Epidemiology of insomnia: what we know and what we still need to learn. Sleep Med Rev. 2002;6:97-111.

2. American Psychiatric Association. Diagnostic and statistical manual of mental disorders. 5th ed. Arlington, VA: APA; 2013. p. 361-422.

3. Sadock B, Sadock V. Kaplan \& Sadock's comprehensive textbook of psychiatry. 
9th ed. Philadelphia, PA: Lippincott Williams \& Wilkins; 2009. p. 2150-77.

4. Sateia MJ. International classification of sleep disorders. 3rd ed. Highlights and Modifications. CHEST. 2014;146(5):1387-94.

5. American Sleep Disorder Association. International classification of sleep disorders. 2nd ed.: Diagnostic coding manual. Westchesler, IL; 2005.

6. Lichstein Kl, Durrence HH, Taylor DJ, et al. Quantitative criteria for insomnia. Behav Res Ter. 2003;41:427-45.

7. Espie CA. Insomnia: conceptual issues in the development, persistence, and treatment of sleep disorder in adults. Annu Rev Psychol. 2002;53:215-43.

8. Petit L, Azad N, Byszewski A, Sarazan F, Power B. Non pharmacological management of primary and secondary insomnia among older people: review of assessment tools and treatments. Age Ageing. 2003;32:19-25.

9. Volltrath. M, Wicky W, Angst J. The Zurich study: VIII Insomnia; Association with depression, anxiety, somatic syndromes, and course of insomnia. Eur Arch Psychiatry Neurol Sci. 1989;239:113232.

10. Silber MH. Chronic insomnia. N Eng J Med. 2005;353:803-10.

11. Viniegra Domínguez MA, et al. Abordaje integrativo del insomnio en atención primaria: medidas no farmacológicas y fitoterapia frente al tratamiento convencional. Aten Primaria. 2015;47(6):351-8. http:// dx.doi.org/10.1016/j.aprim.2014.07.009

12. Buysse D. Insomnia. JAMA. 2013; 309(7):706-16.
13. Mitchell $\mathrm{M}$ et al. Comparative effectiveness of cognitive behavioral therapy for insomnia: a systematic review. BMC Family Practice. 2012;13:40. doi: 10.1186/1471-2296-13-4.

14. Roth T, Drake C. Evolution of insomnia: current status and future directions. Sleep Med. 2004;5(1):23-30.

15. Leger D, Guilleminault C, Dreyfus JP et al. Prevalence of insomnia in a survey of 12778 adults in France. J Sleep Res. 2000;9:35-42.

16. Sateina MJ. Evaluation of chronic insomnia. Sleep. 2000;23:243-63.

17. Ford ES, Wheaton AG, Cunningham TJ, Giles WH, Chapman DP, Croft JB. Trends in outpatient visits for insomnia, sleep apnea, and prescriptions for sleep medications among US adults: findings from the National Ambulatory Medical Care Survey 1999-2010. Sleep. 2014;37(8):1283.

18. Glovinski P, CM Yang, Dubrovsky B, Spielman A. Nonpharmacologic strategies in the management of insomnia:rationale and implementation. Sleep Med Clin. 2008;3:189-204.

19. Thase ME. Correlates and consequences of chronic insomnia. Gen Hos Psychiatry. 2005;27:100-12.

20. Sateia MJ Nowell PD. Insomnia. Lancet. 2004;364:1959-73CM.

21. Glovinski P, CM Yang, Dubrovsky B, Spielman A. Nonpharmacologic strategies in the management of insomnia: rationale and implementtation. Sleep Med Clin. 2008;3:189-204.

22. Yang CM, Spielman A, Glovinski P. Nonpharmacologic strategies in the ma- 
nagement of insomnia. Psychiatr Clin N Am. 2006;29:895-919.

23. Mauss I, Troy A, LeBourgeois M. Poorer sleep quality is associated with lower emotion-regulation ability in a laboratory paradigm. Cogn Emot. 2013;27(3):56776.

24. Stepanski E, Glinn M, Zorick F, et al. Heart rate changes in chronic insomnia. Stress Med. 1994;10:261-6.

25. Bonnet MH, Arand DL. Heart rate variability in insomniacs and matched normal sleepers. Psychosom Med. 1998;60:61015.

26. ML Perlis, DE Guilles, WB Mendelson et al. Psychophysiological insomnia: The behavioural model and a neurocognitive perspective. J Sleep Res. 1997;6:179-88.

27. Johns MW. Relationship between sleep habits, adrenocortical activity and personality. Psychosom Med. 1071;33:499508.

28. Vgontzas AN, Tsigos C, Bixler EO, Lin $\mathrm{HM}$ et al. Chronic insomnia is associated with nyctohemeral activation of the hypothalamic. Pituitary-adrenal axis: Clinical implications. J Clin Endocrinol Metab. 2001;86:3787-94.

29. Bastien $\mathrm{CH}$, Morin $\mathrm{CM}$, Ouellet $\mathrm{MC}$, Blais FC, Bouchard S. Cognitive-behavioral therapy of insomnia: comparison of individual therapy, group therapy, and telephone cosultations. J. Consulting Clin Psychol. 2004;72(4):653-9.

30. Rybarczyk B, Lopez M, Benson R, et al. Efficacy of two behavioral treatment programs for comorbid geriatric insomnia. Psychol Aging. 2002;17:288-98.

31. Hiller RM, Johnston A, Dohnt H, Lovato N, Gradisar M. Assessing cognitive pro- cesses related to insomnia: A review and measurement guide for Harvey's cognitive model for the maintenance of insomnia. Sleep Med Rev. 2015;23:46-53.

32. Harvey L, Inglis SJ, Espie CA. Insomniacs' reported use of CBT components and relationship to long-term clinical outcome. Behav Res Ther. 2002;40:7583.

33. Nofzinger EA, Buysse DJ, Germain A, Price JC, Miedwald JM, Kupfer DJ. Functional imaging evidence for hyperarousal in insomnia. Am J Psychiatry. 2004;161(11):2126-31.

34. Harvey AG, Bélanger L, Talbot L, Eidelman P, Beaulieu-Bonneau S, FortierBrochu É, et al. Comparative efficacy of behavior therapy, cognitive therapy, and cognitive behavior therapy for chronic insomnia: a randomized controlled trial. J Consult Clin Psych. 2014;82 (4):670-83.

35. Perlis M, Aloia M, Millikan A et al. Behavioral treatment of insomnia: A clinical case series study. J Behav Med. 2000;23:149-61.

36. Sutton E. Insomnia. Med Clin N Am. 2014;98:565-81.

37. Sutton E. Psychiatric disorders and sleep issues. Med Clin NAm. 2014;98(5):112343.

38. Hood HK, Rogojanski J, Taryn M. Cognitive-behavioral therapy for chronic insomnia. Curr Treat Options Neurol. 2014; 16:321.

39. Miller CB, Espie CA, Epstein DR, et al. The evidence base of sleep restriction therapy for treating insomnia disorder. Sleep Med Rev. 2014;18(5):415-24.

40. Means MK, Lichstein KL, Epperson MT, et al. Relaxation therapy for insomnia: 
nighttime and day time effects. Behav Res Ther. 2000;38:665-78.

41. Ong JC, Shapiro SL, Manber R. Mindfulness meditation and cognitive behavioral therapy for insomnia a naturalistic 12-month follow-up. Explore. 2009;5:306.

42. Smith MT, Perlis ML, Park A, et al. Comparative meta-analysis of pharmacotherapy and behavior therapy for persistent insomnia. Am J Psychiatry. 2002;159:511.

43. Gancedo-García A, Gutiérrez-Antezana AF, González-García P, Salinas-Herrero S, Prieto Merino D, Suárez-Gil P. Efectividad de una intervención educativa breve en pacientes con insomnio en atención primaria. Aten Primaria. 2014;46(10):549-57.

44. Chennaoui M, Arnal JP, Sauvet F, Léger D. Sleep and exercise: A reciprocal issue? Sleep Med Rev. 2015;20:59-72.

45. Morin CM. Insomnia: Psychological assessment and management. New York: Guillford Press; 1993.

46. Baillargeon L, Landreville P, Verreault $\mathrm{R}$, et al. Discontinuation of benzodiaze- pines among older insomniac adults treated through cognitive-behavioral therapy combined with gradual tapering: A randomized trial. CMAJ. 2003;169:1015-20.

47. Edinger JD, Wohlgemuth WK, Radtke RA, Marsh GR. Dose response effects of behavioral insomnia therapy. Sleep. 2000;23:310.

48. Roth T, Rohers T, Pies R. Insomnia: Patophysiology and implications for treatment. Sleep Med Rev. 2007;10:54153.

49. Edinger JD, Sampson WS. A primary care "friendly" cognitive behavioral insomnia therapy. Sleep. 2003;26:177-82.

50. Taylor DJ, Zimmerman MR, Gardner CE, Williams JM, Grieser EA, Tatum JI, et al. A pilot randomized controlled trial of the effects of cognitive-behavioral therapy for insomnia on sleep and daytime functioning in college students. Behav Ther. 2014;45:376-89.

\section{Correspondencia}

Gabriel Fernando Oviedo Lugo

goviedo@javeriana.edu.co 